\title{
Comparative Evaluation of In-vitro Synergy Testing Methods in Carbapenem-Resistant Acinetobacter Species
}

\author{
Ranu Soni ${ }^{1}$, Varsha Gupta ${ }^{1}$, Satinder Gombar ${ }^{2}$, Jagdish Chander $^{1}$, Priya Datta ${ }^{1}$ \\ ${ }^{1}$ Department of Microbiology, Government Medical College Hospital, Chandigarh, India \\ ${ }^{2}$ Department of Anaesthesia \& Critical care, Government Medical College Hospital, Chandigarh, India
}

\begin{abstract}
Objective: There is an increasing incidence of Acinetobacter species causing serious hospital acquired infections such as blood stream infections (BSI), catheter associated urinary tract infections (CAUTI) and lower respiratory tract infections(LRTI) with high mortality rate of $30-75 \%$ in patients having long hospital stay especially in critical care units (CCUs). During the past decade, multi drug resistant Acinetobacter isolates have presented a real challenge to clinicians and are posing difficulties in treatment. Carbapenem-resistant Acinetobacter species particularly $A$. baumannii, represent a growing public health concern, since they often confer resistance to other critically important antimicrobials.
\end{abstract}

Methodology: In the present study, various antibiotic combinations such as colistin + meropenem, imipenem + tigecycline, polymyxin B + azithromycin and doripenem + sulbactam are used for MDR Acinetobacter infections and tested for in-vitro combination test by Epsilometric test and Broth Micro dilution and confirmation done by time kill assay.

Results: We found synergistic results with all three methods used in study with three combinations significantly $(p<0.05)$ but with one combination i.e. doripenem+sulbactam, synergy found only with Etest not with MCB and time kill assay (p 0.069).

Conclusions: We have also observed and compared outcome of the patients with respect to the treatment received during hospital stay, but we did not found significant difference between two groups, (two drug regimen \& three drug regimen) regarding their mortality. J Microbiol Infect Dis 2019; 9(1): 23-33.

Keywords: Acinetobacter species, drug synergism, antibiotic sensitivity testing

\section{INTRODUCTION}

There is an increasing incidence of Acinetobacter species causing serious hospital acquired infections such as blood stream infections(BSIs), catheter associated urinary tract infections(CAUTIs), lower respiratory tract infections(LRTIs) and skin \& soft tissue infections with high mortality rate of $30-75 \%$ in patients with long hospital stay especially in critical care units (CCUs) [1].

With the rise of incidence of carbapenemresistant Acinetobacter species, there is critical need to discover safe and effective therapeutic strategies. Apart from colistin the treatment options left to treat carbapenem-resistant Acinetobacter species are tigecycline, polymyxin $\mathrm{B}$, fosfomycin, sulbactam as monotherapy [2].
Usage of polymyxins has been increasing in trends, the only therapeutic options for these carbapenem-resistant Acinetobacter species infections. The limitations related to polymyxins usage are irrelevant dosage, promising adverse effects (nephrotoxicity) and emergence of resistance [2]

As various therapeutic approaches have been contemplated like combination therapy through in-vitro ( in laboratory), in-vivo ( animal model) and clinical trials, should be included in therapeutic regime as the fortitude for cure of infections caused by isolates which are colistinsusceptible as well as colistin-resistant [3].

In this present study, we have used four antibiotic combinations with three different methods for Carbapenem-resistant

Correspondence: Dr. Ranu Soni, Department of Microbiology, Government Medical College Hospital, Chandigarh, India 
Acinetobacter species infections. Although only a few study have been done in India on synergy, Anandan et al, from CMC Vellore has published in-vitro synergy based on colistin combination with sulbactam and meropenem on MDR Acinetobacter species isoated from ventilator associated pneumonia. This study showed commendable synergy by time kill analysis with combination of sulbactam \& meropenem. [4] Our study would help us to illustrate the appropriateness of synergy testing to anticipate the action of specific antimicrobials combinations against carbapenem-resistant Acinetobacter species, which can be further used by clinicians in treatment.

\section{METHODS}

The study was planned and experimental conducted from January 2016 to June 2017 in the department of Microbiology, Government Medical College \& Hospital, Chandigarh, India. A total of 100 non repeated strains of Acinetobacter species were isolated from the various samples received from ICU \& wards of our tertiary care hospital. Ethical clearance was taken from the Ethical committee of Govt. Medical College and Hospital, Chandigarh.

\section{Isolation and susceptibility testing}

Acinetobacter species was identified using standard microbiological methods. Antimicrobial susceptibility testing of Acinetobacter isolates was done using Kirby Bauer disk diffusion method according to Clinical Laboratory Standards Institute (CLSI) guidelines 2016 [5].

\section{MIC determination}

Additionally for all those imipenem resistant isolates by disk diffusion method, MIC values were determined by Etest (Bio Merieux) and then Broth Microdilution Method (BMD). The MIC determination was done for the following antimicrobials used in combinations in our studycolistin, meropenem, tigecycline, imipenem, polymyxin B, azithromycin, doripenem, sulbactam. The antimicrobials labeled as resistant/sensitive based on their breakpoints given by CLSI 2016. [5] No CLSI, European Committee on Antimicrobial Susceptibility Testing (EUCAST) and United States of Food and Drug Administration (USFDA) breakpoints are there for tigecycline, sulbactam and azithromycin for Acinetobacter so in our study we used Enterobacteriaceae breakpoints given by British Society for Antimicrobial Chemotherapy (BSAC) [6].

\section{Synergy testing}

All carbapenem (imipenem) resistant Acinetobacter species isolates were tested for in-vitro synergy testing of various AMAs combinations by practicing Etest, Broth Microdilution method (BMD) and Time Kill Analysis (TKA) as gold standard.

The various AMAs combinations tested were:

a) Colistin + meropenem (Sigma-Aldrich, USA)

b) Tigecycline + imipenem (Sigma-Aldrich, USA)

c) Polymyxin B + azithromycin (Sigma-Aldrich, USA)

d) Sulbactam + doripenem.( Sigma-Aldrich, USA)

\section{Synergy testing by Epsilometric test, (Etest)}

Mueller-Hinton Agar (MHA, Hi Media) was inoculated with $0.5 \mathrm{Mc}$ Farland suspensions of study isolates. This method was performed conceding to the method interpreted by Balaji V., et al, the fixed ratio Epsilometric method [2].

Interpretation: The interpretation of AMA combinations methods were determined by observing the MIC of individual drugs; drug A, which were active drugs in combinations(colistin, tigecycline, sulbactam and polymyxin B) and the MIC of drug B, which were resistant for the study isolates (meropenem, imipenem, doripenem, and azithromycin) after prediffusion of active drug in the medium. Then synergy was determined as follows by combined MIC by the formula [7].

If MIC of combined drug $A+B$ is reduced by 3 fold dilutions as compared to MIC of drug A (active) the result was considered as 'Synergistic'.

\section{Synergy testing by Broth Micro Dilution Checker-Board (MBD)}

Test methods are based on standard CLSI M7A8 broth dilution susceptibility methods [8]. Antimicrobial stock solutions were prepared according to manual given in CLSI M7-A8 in the appropriate diluents as indicated in CLSI [5]. 
Number of Concentrations tested prepared according to requirement for each combinations. [9] Preparation of checkerboard micro dilution panel was done according to CLSI M7-A8 [8]. Inoculums was prepared matched with optical density 0.5 Mc Farland. The MIC tray was incubated at $35+/-2^{0} \mathrm{C}$. Reading was taken as MIC of single drug alone and in combination after $24 \mathrm{hrs}$. incubation.

\section{Synergy interpretation by Broth micro dilution method}

The Fractional Inhibitory Concentration Index (FICl) was estimated to determine synergy by implicating subsequent equation ${ }^{[7]}$

$\mathrm{FICl}=\mathrm{FIC}_{\mathrm{A}}+\mathrm{FIC}_{\mathrm{B}}$

$\mathrm{FICI}=\frac{\text { MICAB }}{\text { MICA }}+\frac{\text { MICBA }}{\text { MICB }}$

FIC $_{A}$ : Fractional Inhibitory Concentration (FIC) of drug $A$,

$\mathrm{FIC}_{\mathrm{B}}$ : FIC of drug B

$\mathrm{MIC}_{\mathrm{AB}}$ : Minimum Inhibitory Concentration (MIC) of drug $A$ in presence of drug $B$

$\mathrm{MIC}_{\mathrm{BA}}-\mathrm{MIC}$ of drug $\mathrm{B}$ in presence of drug $\mathrm{A}$

$\mathrm{MIC}_{\mathrm{A}}-\mathrm{MIC}$ of individual drug $\mathrm{A}$

$\mathrm{MIC}_{\mathrm{B}}-\mathrm{MIC}$ of drug $\mathrm{B}$ alone

Synergy will be interpreted by [10] -

\section{Interpretation}

$\mathrm{FICl} \leq 0.5 \rightarrow$ Synergy,

$\mathrm{FICl}>0.5$ to $\leq 1 \rightarrow$ addition,

$\mathrm{FICl}>1$ to $\leq 4 \rightarrow$ Indifference,

$\mathrm{FICl}>4 \rightarrow$ Antagonism

\section{Synergy testing by Time-kill Analysis}

To perform the time-kill assay, there are various specifications must be known. We calculated the MIC of the each antimicrobial drug in combination for the Acinetobacter isolates of interest by a Broth Micro dilution method [9].

\section{Procedure}

The procedure was followed according to Microbiological Techniques \& methodsLaboratory Manuals, Clinical Microbiological Procedures, H.D Isenberg.[9] Inoculum matched $0.5 \mathrm{McF}$ arland was prepared and added to glass tube containing single drug or combined drugs and incubated them at $37^{\circ} \mathrm{C}$ with intermittent shaking for different timings and then sub cultured with their respective timings i.e. ' 0 ' hour, ' 4 ' hours, ' 6 ' hours, ' 12 ' hours.

\section{Interpretation}

Results were interpreted as individual agent MIC and combined MICs as follows [9]:

We determined the log $10 \mathrm{CFU} / \mathrm{ml}$ for individual agents and the decreased colony counts were compared with the growth control at sample times. Then we determined the difference in $\log 10$ Colony forming Unit (CFU) /ml between the most vital antimicrobial drug individually at $1 / 4$ th MIC and the combination of drugs at $1 / 4$ th or $1 / 8$ th MIC. Synergism is usually defined as decrease in Colony forming units by more than $2-\log 10 \mathrm{CFU} / \mathrm{ml}$ by the combination of drugs as compared with the most active individual drug.

\section{Quality control strains}

For MIC assurance quality control strains were included in the study; Escherichia coli (ATCC 25922) and Pseudomonas aeruginosa (ATCC 27853).

In addition to microbiological testing the clinical data of the patients whose strains are included in the study have been recorded.

\section{RESULTS}

A total of 100 non repeated strains of Acinetobacter species were isolated from various clinical samples (blood culture, pus aspirate, Bronco-alveolar lavage, ascitic fluid, tracheal aspiration, tissue, high vaginal swab) during study period. The Acinetobacter species were identified on the basis of colony characteristics, staining properties and biochemical reactions.

\section{Antibiotic susceptibility}

The observations were made on antimicrobial sensitivity methods using Kirby Bauer diskdiffusion method for the drugs advocated by CLSI 2016(Table 1). To ascertain the breakpoints for these Acinetobacter isolates CLSI guidelines-2016 were referred, BSAC for tigecycline and sulbactam as no CLSI breakpoints (Table 2) are given for these drugs and other research studies have done the same. 


\section{MIC determination}

MIC determination was done using Etest and then Broth Microdilution method (BMD) for all the antimicrobial agents used in the study.

\section{MIC dtermination by Etest}

MIC was determined using Etest for individual drugs used in combination shown in (Table 3). Most of the strains showed high MIC values (resistant) for meropenem, imipenem, doripenem and azithromycin.

\section{MIC determination by BMD method}

MIC determination was also done by BMD method for all drugs used in the study because MIC determined by Etest was not reliable and upto the mark as a limited MIC values are graded on the strips which can be calculated \& for further reading of MIC values BMD method is more reliable as the accurate value can be calculated by it (Table 4).

\section{Synergy testing}

Results with different combinations, using three methods Etest, BMD and the gold standard TKA are as follows;

\section{Synergy testing interpretation using Etest with all four combinations}

Firstly synergy testing by Etest with different antibiotics combinations showed different interactions on hundred Acinetobacter isolates (Table 5) showing Etest with meropenem and colistin combination.

\section{Synergy testing interpretation using BMD with all four combinations;}

The resultant interpretation of BMD method was done using Fractional Inhibitory Concentration Values $(\mathrm{FICl})$ for each isolates with all combinations [7]. It was found that with the combination meropenem+colistin, second combination imipenem+ tigecycline and third combination azithromycin+ polymyxin B showed synergistic results but with the forth combination doripenem+ sulbactam, none of the isolate showed synergy (Table 6).

\section{Synergy testing interpretation using time kill assay with all four combinations;}

In our study, TKA was performed only on those Acinetobacter isolates, which showed synergy with both Etest and Broth Micro dilution method. It was found that with the combination of meropenem + colistin, $53 \%$ isolates showed synergy. The second combination imipenem+ tigecycline, $52 \%$ isolates showed synergy. Third combination azithromycin + polymyxin B, 48\% isolates showed synergy at different timings with respective MICs.

With the combination of doripenem+sulbactam synergy was not detected with any of the isolate. by Broth microdiilution method (Table 7).

\section{Final comparative evaluation between all the four combinations, using different methods Etest, BMD, TKA;}

The results of all four different antimicrobial combinations tested by these three methods.(Table 8) It was found that the results of Etest corroborated with BMD to an extent. We confirmed our results using gold standard test TKA. We found that Acinetobacter isolates showing synergy with both Etest and BMD method, corroborated with TKA results for three combinations and it is $(p<0.05)$ statistically significant for all three combinations with Etest \& BMD but with last combination i.e. doripenem+sulbactam it was not significant statistically $(p=0.069)$; we found synergy with Etest but did not find synergy with both BMD and TKA method (Table 9).

Meropenem \& colistin combination was the best combination amongst all combinations. Comparisons of all four combinations are given in Table 8.

Third combination doripenem + sulbactam showed $86 \%$ synergy with Etest but did not showed synergy by BMD.

\section{Outcome}

The antibiotic regimen given to the patients and their outcome was correlated according to the therapy received. It was just an observation what antibiotics they had recieved during treatment for these carbapenem-resistant Acinetobacter isolates to have an idea that combination therapy or monotherapy which have better outcome.

A total of hundred patients were entailed in our research, those were infected with Acinetobacter species. Consents have been taken before 
incorporate investigation. These patients received distinct combinations of antimicrobials during their hospital stay, some patients recovered successfully and a few died due to worsening of their clinical condition. A total of $41 / 100$ patients received two drug therapies, $19 / 40$ patients recovered successfully, 19/40 died during their therapy and $3 / 40$ patients left against medical advice (LAMA) (Table 10). Out of 100 patients, 59 patients received three drug regimens, in which 28 out of 60 patients recovered with three drug combination therapy, $27 / 60$ of the patients expired and 4/60 patients left against medical advice (Table 10).

Mortality was most commonly associated with patients who have received two drugs regimen of ampicillin-sulbactam, tobramycin \& colistin. Additionally, the consequences of various combinations could be diverge in different survey this may be due to difference in population, diseases, antimicrobial usage, therapeutic options, durations etc.

Table 2. Breakpoint MIC values for antimicrobials used in study[CLSI $\left.-2016^{5,6}\right]$.

\begin{tabular}{lcccc}
\hline Antimicrobials & $\begin{array}{c}\text { MIC value } \\
\text { Sensitive }\end{array}$ & $\begin{array}{c}\text { MIC value } \\
\text { Resistant }\end{array}$ & $\begin{array}{c}\text { Zone diameter } \\
\text { (Sensitive) }\end{array}$ & $\begin{array}{c}\text { Zone diameter } \\
\text { (Resistant) }\end{array}$ \\
\hline Meropenem $(10 \mu \mathrm{g})$ & $\leq 2$ & $\geq 8$ & $\geq 18$ & $<14$ \\
Imipenem & $\leq 2$ & $\geq 8$ & $\geq 22$ & $<18$ \\
Doripenem & $\leq 2$ & $\geq 8$ & $\geq 18$ & $<14$ \\
Sulbactam & $\leq 4$ & $\geq 16$ & & \\
Azithromycin & - & - & - & - \\
Tigecycline & $\leq 2$ & $\geq 8$ & - & $\leq 10$ \\
Colistin & $\leq 2$ & $\geq 4$ & $\geq 11$ & $<11$ \\
Polymyxin B & $\leq 2$ & $\geq 4$ & $\geq 12$ & \\
\hline
\end{tabular}

Table 3: MIC range of individual antibiotics used in combinations determined by Etest

\begin{tabular}{lcccccccc}
\hline MIC range (mg/L) & MER (n) & IMP (n) & DOR (n) & AZI (n) & COL (n) & TIG (n) & SUL (n) & PB (n) \\
\hline $0-2$ & 1 & 1 & 1 & 2 & 95 & 100 & 1 & 100 \\
$2-8$ & 0 & 0 & 2 & - & 5 & - & 42 & - \\
$8-32$ & 25 & 6 & 46 & - & - & - & 57 & - \\
$>32$ & 74 & 93 & 51 & - & - & - & - & - \\
$32-64$ & - & - & - & 4 & - & - & - & - \\
$64-128$ & - & - & - & 25 & - & - & - & - \\
$128-256$ & - & - & - & 69 & - & - & - & - \\
\hline
\end{tabular}

MER=Meropenem, IMP=Imipenem, DOR-Doripenem, AZI=Azithromycin, COL=Colistin, TIG=Tigecycline, SUL=Sulbactam, PBPolymyxin $B, n=$ number of isolates 
Table 4: MIC range of individual antibiotics with Broth Micro dilution method for drugs used in combinations.

\begin{tabular}{lcccccccc}
\hline $\begin{array}{l}\text { MIC range } \\
(\mathbf{m g} / \mathbf{L})\end{array}$ & MER (n) & IMP (n) & DOR (n) & AZI (n) & COL (n) & TIG (n) & SUL (n) & PB (n) \\
\hline $0-2$ & 1 & 1 & 0 & 2 & 88 & 100 & 1 & -38 \\
$2-8$ & 0 & 0 & 2 & 0 & 12 & - & - & - \\
$8-32$ & 15 & 21 & 48 & 0 & - & - & - & - \\
$32-64$ & 55 & 62 & 36 & 12 & - & - & - & - \\
$64-128$ & 16 & 15 & 14 & 25 & - & - & -
\end{tabular}

MER=Meropenem, IMP=Imipenem, DOR-Doripenem, AZI=Azithromycin, COL=Colistin, TIG=Tigecycline, SUL=Sulbactam, PBPolymyxinB, $n=$ number of isolates

Table 5: Synergy test results of four different antibiotics combination using Etest strip in Carbapenem-resistant Acinetobacter isolates.

\begin{tabular}{|c|c|c|c|c|}
\hline Antibiotic combinations & Synergy (n) & Indifference (n) & Addition (n) & Antagonism (n) \\
\hline Meropenem + Colistin & 89 & No & 11 & No \\
\hline Azithromycin+ Polymyxin B & 85 & No & 15 & No \\
\hline Imipenem+Tigecycline & 82 & 2 & 16 & No \\
\hline Doripenem +Sulbactam & 86 & 2 & 12 & No \\
\hline
\end{tabular}

$\mathrm{n}=$ number of isolates

Table 6. Results of synergy testing of four different antibiotic combinations with Broth Micro dilution checkerboard method

\begin{tabular}{lcccc}
\hline Antibiotic combinations & Synergy (n) & Indifference (n) & Addition (n) & Antagonism (n) \\
\hline Meropenem +Colistin & 54 & 31 & 15 & - \\
Imipenem +Tigecycline & 52 & 32 & 16 & - \\
Azithromycin + Polymyxin B & 48 & 23 & 29 & - \\
Doripenem +Sulbactam & - & 64 & 36 & - \\
\hline
\end{tabular}

$\mathrm{n}=$ number of isolates

Table 7: Synergy testing with Time kill assay of Acinetobacter isolates showing Synergy with both Broth micro dilution and Etest with four antibiotics.

\begin{tabular}{lccc}
\hline Antibiotic combinations & $\begin{array}{l}\text { Synergy with } 1 / 2 \text { MIC } \\
\text { (at 4, 6, 12 hours), } \mathbf{n}\end{array}$ & $\begin{array}{l}\text { Synergy with } 1 / 2 \text { MIC } \\
\text { (at 4, 6, 12 hours), } \mathbf{n}\end{array}$ & $\begin{array}{c}\text { Synergy with } 1 / 2 \text { MIC } \\
\text { (at 4, 6, 12 hours), } \mathbf{n}\end{array}$ \\
\hline Meropenem +Colistin & 53 & 53 & 53 \\
Azithromycin+ Polymyxin B & 48 & 48 & 48 \\
Imipenem +Tigecycline & 52 & 52 & 52 \\
Doripenem +Sulbactam & SND & SND & SND \\
\hline SND=Synergy Not Detected & & &
\end{tabular}


Table 8. Comparison between Etest, MCB, TKA results.

\begin{tabular}{lccccccccc}
\hline \multirow{2}{*}{ AMA combination } & \multicolumn{3}{c}{ Etest (n) } & \multicolumn{3}{c}{ MCB (n) } & \multicolumn{3}{c}{ TKA (n) } \\
\cline { 2 - 9 } & S & I & A & S & I & A & S & I & A \\
\hline Mero+ Col & 89 & - & 11 & 54 & 31 & 15 & $53-$ & - & - \\
Imp+ Tige & 82 & 2 & 16 & 52 & 32 & 16 & 52 & - & - \\
Azi+ PB & 85 & - & 15 & 48 & 23 & 29 & 48 & - & - \\
& & & & & & & & - & -
\end{tabular}

$\mathrm{S}=$ Synergy, I=Indifference, A=Antagonism, MER=Meropenem, IMP=Imipenem, DOR-Doripenem, AZI=Azithromycin, COL=Colistin, TIG=Tigecycline SUL=Sulbactam, PB- PolymyxinB

Table 9. Table of Significance.

\begin{tabular}{|c|c|c|c|c|}
\hline \multirow{2}{*}{$\begin{array}{l}\text { Variables } \\
\text { Synergy }\end{array}$} & \multirow[t]{2}{*}{$\mathbf{N}$ (Isolates) } & \multicolumn{2}{|c|}{$\mathrm{FICl}$} & \multirow[t]{2}{*}{ ANOVA; $p$} \\
\hline & & Mean & SD & \\
\hline Meropenem+ colistin & 54 & 0.38 & 0.11 & $33.2 ;<0.00$ \\
\hline Imipenem + tigecycline & 52 & 0.48 & 0.04 & \\
\hline Azithromycin +polymyxin B & 48 & 0.48 & 0.04 & \\
\hline
\end{tabular}

Table 10. Comparison of Two \& Three drug regimen, patients received in ICU and outcome of patients.

\begin{tabular}{|c|c|c|c|c|c|}
\hline $\begin{array}{l}\text { Antibiotics } \\
\text { (Two drug } \\
\text { regimen) }\end{array}$ & $\begin{array}{l}\text { Recovered } \\
\text { patients } \\
\text { (n) }\end{array}$ & $\begin{array}{l}\text { Expired } \\
\text { patients } \\
\text { ( } \mathrm{N}=\text { no. of } \\
\text { cases) }\end{array}$ & $\begin{array}{c}\text { Antibiotics } \\
\text { Three drug } \\
\text { regimen }\end{array}$ & $\begin{array}{c}\text { Recovered patients } \\
\text { ( } \mathrm{N}=\text { no. of cases) }\end{array}$ & $\begin{array}{l}\text { Expired patients } \\
\text { ( } \mathrm{N}=\text { no. of cases) }\end{array}$ \\
\hline I \& CL & 11 & 5 & $\mathrm{I}+\mathrm{MR}+\mathrm{CL}$ & 3 & - \\
\hline$M R \& C L$ & 6 & - & $\mathrm{AS}+\mathrm{I}+\mathrm{CL}$ & 11 & 6 \\
\hline I \& MR & 1 & - & $\mathrm{AS}+\mathrm{TB}+\mathrm{CL}$ & - & 7 \\
\hline PT \& I & - & 5 & $\mathrm{AK}+\mathrm{I}+\mathrm{CL}$ & 4 & 4 \\
\hline $\mathrm{PT} \& \mathrm{CL}$ & - & 3 & $\mathrm{PT}+\mathrm{I}+\mathrm{CL}$ & 8 & 3 \\
\hline PT \& CF & 1 & - & $\mathrm{AK}+\mathrm{TB}+\mathrm{I}$ & - & 5 \\
\hline AS \& I & - & 2 & $\mathrm{AK}+\mathrm{MR}+\mathrm{CL}$ & 2 & - \\
\hline$A S \& C L$ & - & 2 & $\mathrm{AK}+\mathrm{AS}+\mathrm{CL}$ & - & 1 \\
\hline I \& CF & - & 1 & $\mathrm{AS}+\mathrm{PT}+\mathrm{CL}$ & - & 1 \\
\hline $\mathrm{TB}+\mathrm{CL}$ & - & 1 & $\mathrm{AS}+\mathrm{I}+\mathrm{CF}$ & - & - \\
\hline Total & 19 & 19 & Total & 28 & 27 \\
\hline
\end{tabular}

$\mathrm{n}=$ No. of cases, $\mathrm{I}=$ imipenem, $\mathrm{MR}=$ Meropenem, $\mathrm{CL}=$ Colistin, $\mathrm{CF}=$ Ciprofloxacin, $\mathrm{PT}=$ Piperacillin-Tazobactam, AS=Ampicillin-Sulbactam, TB=Tobramycin, AK=Amikacin

\section{DISCUSSION}

In the last few years, carbapenem-resistant Acinetobacter species has been labeled as a "red alert" human microorganism, triggering distress amongst the health care professionals, due to its emergence of antibiotic resistance pattern [10].

Over the last decade in Europe and worldwide, Acinetobacter species especially Acinetobacter baumannii has become increasingly resistant to carbapenems drugs, a broad spectrum beta- lactam group of antibiotics. It is an important therapeutic option for patients infected with multi drug-resistant gram-negative bacteria [11].

There are several reasons that reinforce the need of combination regimen, are microbiological synergy between different groups of antibiotics. This strategy would help both microbiologist and clinician to select the treatment regimen for the patients infected with MDR Acinetobacter and other gram negative organisms. Our strategies are directed to improve survival of patients, better clinical 
outcomes and prevention of the emergence of resistant Acinetobacter species strains.

In our research the first, colistin-meropenem combination revealed commendable synergy by using Etest, BMD method which was confirmed by TKA. It was found that all the isolates showed synergy with TKA and synergy with this combination was statistically convincing $(p<0.05)$.

An analysis done in the Medical Center, Beirut, manifested that the combination experiments between colistin \& carbapenem showed very high degree of addition by using methods used in our study (Etest, BMD, TKA) [12]. Another study conducted by LeMinh et al, in Vietnam, showed synergistic rates with colistinmeropenem and colistin-imipenem respectively $68 \%$ and $36 \%$ [13]. Justin RL, showed the combination with colistin \& carbapenems has been studied earlier where authors conveyed that microorganism re-growth can be stopped or regulated. The resultant MIC of colistin also decreased by two-fold. This is very much suggestive of using lower dosage of colistin in treatment with minimal of adverse effects. Also, above study revealed significantly additive effect of colistin and carbapenem combinations ( $p<$ 0.05) [14].

The second combination used in our study i.e. imipenem \& tigecycline showed a good synergistic effect by all the three methods described in study. The result was significant statistically $(p<0.05)$. So the combination of tigecycline+imipenem is also a good choice for carbapenem-resistant Acinetobacter isolates.

A study performed in-vitro synergy test by D'Arezzo et al, showed that by using BMD and TKA methods with the combination of imipenem + tigecycline, found synergistic interaction (MICs for tigecycline $(0.5 \mathrm{mg} / \mathrm{L})$ and imipenem(16 $\mathrm{mg} / \mathrm{L})$, respectively) in Acinetobacter species isolates [15]. The author concluded that, combination therapy with tigecycline \& imipenem could depicted a better regimen to fight with resistant strains to both these drugs individually. Another study done by Kuo \& Lee et al showed that combination therapy (imipenem + tigecycline) possesses synergistic activity that is effect could be more than addition of two antimicrobials individually. This effect has been shown to increase positive outcomes in the treatment of immunocompromised patients infected with Carbapenem-Resistant Acinetobacter (CRA)-related infections [16,17]. Pachon Ibanez et al, showed that tigecycline is a bacteriostatic agent, used to treat CRA infections and tigecycline was more active than imipenem against CRA. The author has concluded that to ameliorate the clinical condition of a patient tigecycline is suitable to combine with another antimicrobial drug like carbapenems [18].

To culminate the results of in-vitro study combination of carbapenem and tigecycline can be included in therapeutic options and assure to be advantageous for the cure of CRA infections.

In concern of third combination azithromycin \& polymyxin $B$, our study showed good results by all the three methods. By using Etest, and BMD method this combination also showed synergy TKA was done to confirm the synergistic results and displayed $100 \%$ corroborative results with BMD method. The resultant synergy with both of these methods showed statistically significance $(p<0.05)$. A study done by Nageeb et al, detected synergy by experimenting Epsilometric test between polymyxin and azithromycin (100\% isolates) and in $66 \%$ isolates it was done by TKA with the same combination. Author concluded that azithromycin and polymyxin B combination is justifiable and advised. It may be converted into convenient curative strategy by synergism between two or more drugs results in lowering the effective MIC values with addition to their pharmacological and biological effects to determine the actual therapeutic dosage of these antimicrobial in combinations for clinical practice [19]. Manikal et al interrogated synergism method i.e. Broth micro-dilution with polymyxin and azithromycin combinations in contrary to Acinetobacter isolates belonging to distinct genotypes. They found approximately $80 \%$ resultant synergy with this combination and $20 \%$ isolates showed addition effect. Almost $8 \%$ of Acinetobacter isolates showing synergy with azithromycin and polymyxin combination were resistant to polymyxin [20]. Luber et al, conveyed that with Broth microdilution method, moreover the results were in favor of addition compared to Epsilometric method $(p<0.05)$. This could be due to disparity in classical property between the liquid and the solid media that were 
used for these experiments in Broth microdilution and Etest respectively [21].

In consideration to last combination i.e. doripenem and sulbactam, in our study we found synergy with doripenem and sulbactam combination by using Etest only but did not find synergy with MCB and time kill assay. The resultant synergism was not showed analytically significant $(p=0.069)$. Elucidation could be that all study isolates in our study had higher MIC values for both sulbactam and doripenem so could not interact with each other in the effective way. As none of the drug contributed an active role for this combination against Acinetobacter isolates which was used in the study.

Studies favoring our results regarding this combination showed wide variations. A study performed in-vitro synergy testing by Dinc et al, using combination of doripenem with different antibiotics including sulbactam, colistin, tigecycline, amikacin. In this study, it was clarified that the combination strategy had an improved effect to clear bacterial growth compare to doripenem drug therapy alone. They used different antibiotic combinations against Acinetobacter baumannii, in their study. Combination therapy with doripenem \& tigecycline had more rapid effect to inhibit bacterial growth [22]. Pongpech et al. experimented on CRAB and multi-drug resistant $A$. baumannii isolates with multidrug therapy using sulbactam. $\mathrm{He}$ and his colleagues reported synergistic effects using sulbactam \& carbapenem $(70 \%)$ or sulbactam \& colistin (53\%) respectively [23].

There are several studies which reveal synergistic effects with combination of sulbactam \& carbapenems. In our study we have chosen doripenem \& sulbactam combination. This can be elucidated by that doripenem is a comparatively newly advertised carbapenem group of drug with an in-vitro activity against both group of microorganisms (gram-positive \& gram-negative microorganisms). It is more reliable than other carbapenems [24]. The second drug, sulbactam was chosen as active drug because it expressed intrinsic bactericidal activity against MDR Acinetobacter isolates infections, as it inhibits the penicillin- binding proteins [25]. It is irreversible inhibitor of beta-lactamase producing organisms like Acinetobacter and does not allow enzymes to degrade the antibiotic [26].

Related to outcome of patients, various studies across the world and few studies from India, suggested that combination therapy has several advantages over monotherapy with regard to mortality of critically ill patients [27].

In our study there is not disagreement between both the groups with respect to their mortality ( $46 \%$ in double drug therapy vs. $45 \%$ in triple drug therapy). Since none of the patient in our study received monotherapy, therefore we could not compare monotherapy $\mathrm{v} / \mathrm{s}$ combination therapy in regard to mortality of the patients. Hence probable reasons why even after giving combination therapy the patients had higher mortality were delayed appropriate antibiotic therapy, severity of clinical illness and associated co-morbidities like diabetes, hypertension, acute renal failure, cardiac disease and cancer.

Falagas et al., proposed a methodical review that combination therapy has offer a great correlative asset over single drug therapy clinically in survival of patients infected with carbapenem-resistant gram negative organisms [28]. An observational study done by Ghafur et al, from India, found that there was no remarkable distinction in mortality between single drug and two or three drug combinations in the patients infected with Acinetobacter and Pseudomonas bacteremia $(n=36)$ as well $(P=$ 0.067 ), except in neutropenic patients, where combination therapy accomplished better outcome [29].

Due to recurrent modification of antimicrobial treatment, outcome of this population might be challenging to assess the severely ill patients included in these studies. Besides, the results for specific combinations might vary among different studies due to dissimilarities in population of patients, co morbidities, treatment regime, dosage, durations, and strain-dependent factors. This data was just to have an idea that the clinician are using which two and three drug combinations more frequently and then once our results are available in formulating antibiotic policy for the Institute. In-vitro analysis of data suggests that combination therapeutic strategy can be adequate when microorganisms are 
found to be resistant to single drugs individually. Variations were found between different studies.

Another review by Lisa Saiman, recommended, that in clinical practice, clinician start empirical antibiotic therapy before getting the result of antimicrobial sensitivity. If patient responded to that drug clinically, clinician would continue it. This is the promising implication for synergy testing and is applicable when a patient is failing to respond clinically to the treatment. However, synergy testing can be advisable to decide the treatment when a patient is not responding to antimicrobial agents, while the microorganisms are susceptible [30].

To treat such infection caused by carbapenemresistant microorganisms, this study and analysis provide us navigation. To boost up the clinical events of these patients, we have a devious way to go, to ameliorate outcomes further.

The constraint of our study is that combination effects detected by Broth Micro dilution \& time kill assay could not be analyzed with molecular methods. The mechanisms of resistance were not studied.

\section{ACKNOWLEDGMENTS}

Funding: This study was supported by internal funding.

Transparency declaration: None to declare.

\section{REFERENCES}

1. Bergogne-Bérézin E, Towner KJ. Acinetobacter spp. as nosocomial pathogens: Microbiological, clinical, and epidemiological features. Clin Microbiol Rev 1996; 9:148-165.

2. Balaji V, Jeremiah SS, Baliga PR. Polymyxins: Antimicrobial susceptibility concerns and therapeutic options. Indian J Med Microbiol 2011; 29:230-242.

3. Doi Y, Murray GL, Peleg AY. Acinetobacter baumannii: evolution of antimicrobial resistancetreatment options. Semin Respir Crit Care Med 2015; 36:85-98.

4. Anandan S, Jennifer L, Pragasam AK, et al Synergy Testing between Sulbactam and Meropenem/ Colistin in MDR Acinetobacter baumannii-calcoaceticus Complex Isolated from Ventilator Associated Pneumonia. J Infect Dis Ther 2016; 4:299.

5. Clinical and Laboratory Standards Institute.2016. Performance Standards for Antimicrobial
Susceptibility Testing; approved standard. $26^{\text {th }}$ ed. supplement CLSI document M100S. Clinical and Laboratory Standards Institute Wayne, PA, USA.

6. Andrews JM. BSAC Standardized disc susceptibility testing methods version $6 . \mathrm{J}$ Antimicrob Chemother 2007; 60:20-41.

7. Sopirala MM, Mangino JE, Gaberyes WA, et al. Synergy testing by Etest, microdilution checkerboard, and time-kill methods for pan-drugresistant Acinetobacter baumannii. J Antimicrob Chemother 2010; 54:4678-4683.

8. Clinical and Laboratory Standards Institute.2009. Methods for dilution antimicrobial susceptibility tests for bacteria that grow aerobically; approved standard. 8th ed. Supplement CLSI document M07A8. Clinical and Laboratory Standards Institute Wayne, PA, USA.

9. Garcia, Lynne Shore, Isenberg, Henery D. Microbiological Techniques \& methods-Laboratory Manuals. Clinical Microbiology Procedures Handbook- $3^{\text {rd }}$ edition, 2007 update Washington, 2007; 2:5.18.1-14.

10. Howard A, O'Donoghue M, Feeney A, Sleator RD. Acinetobacter baumannii An emerging opportunistic pathogen, Virulence 2012; 3:243-250.

11. Higgins PG, Dammhayn C, Hackel M, Seifert H. Global spread of carbapenem-resistant Acinetobacter baumannii. J Antimicrob Chemother 2010; 65:233-238.

12. Soudeiha MAH, Dahdouh EA, Azar E, Sarkis DK, Daoud Z. In vitro Evaluation of the ColistinCarbapenem Combination in Clinical Isolates of $A$. baumannii, Using the Checkerboard, Etest, and Time-Kill Curve Techniques. Front Cell Infect Microbiol 2017; 7:209.

13. LeMinh V, Nhu NTK, Phat VV, et al. In vitro activity of colistin in antimicrobial combination against carbapenem-resistant Acinetobacter baumannii isolated from patients with ventilatorassociated pneumonia in Vietnam. J Med Microbiol 2015; 64:1162-9.

14. Justin RL, Roger LN, Brian TT. Synergistic combinations of polymyxins. Int J Antimicrob Agents 2016; 48:607-613.

15. D'Arezzo S, Capone A, Petrosillo N, et al. Epidemic multidrug resistant Acinetobacter baumannii related to European clonal types I and II in Rome (Italy). Clin Microbiol Infect 2009; 15:347357.

16. Kuo LC, Yu CJ, Kuo ML, Chen WN, Chang CK, Lin HI. Antimicrobial resistance of bacterial isolates from respiratory care wards in Taiwan: A horizontal surveillance study. Int J Antimicrob Agents 2008; 31:420-426.

17. Lee NY, Wang CL, Chuang YC, Yu WL, Lee HC, Chang CM. Combination carbapenem- 
ampicillin/sulbactam therapy for critically ill patients with multidrug-resistant Acinetobacter baumannii bacteremia: Four case reports and an in vitro combination synergy study. Pharmacotherapy. 2007; 27:1506-1511.

18. Pachon-lbanez ME, Jimenez-Mejias ME, Pichardo C, Lianos AC, Pachon J. Activity of tigecycline (GAR-936) against Acinetobacter baumannii strains, including those resistant to imipenem. Antimicrob Agents Chemother 2004; 48:4479-4481.

19. Nageeb W, Metwally L, Kamel M, Zakaria S. In vitro antimicrobial synergy studies of carbapenemresistant Acinetobacter baumannii isolated from intensive care unit of tertiary care hospital in Egypt $\mathrm{J}$ Infect public Health 2015; 8:593-602.

20. Manikal VM, Landman D, Saurina G, Oydna E, Lal $\mathrm{H}$, Quale J. Endemic carbapenem-resistant Acinetobacter species in Brooklyn, New York: citywide prevalence, inter-institutional spread, and relation to antibiotic usage. Clin Infect Dis 2000; 31:101-106.

21. Luber P, Bartelt,E, Genschow E, Wagner J, Hahn $\mathrm{H}$. Comparison of broth microdilution, E Test, and agar dilution methods for antibiotic susceptibility testing of Campylobacter jejuni and Campylobacter coli. J Clin Microbiol 2003; 41:1062-1068.

22. Dinc G, Demiraslan H, Elmali F, Ahmed SS, Alp E, Doganay M. Antimicrobial efficacy of doripenem and its combinations with sulbactam, amikacin, colistin, tigecycline in experimental sepsis of carbapenemresistant Acinetobacter baumannii. New Microbiol 2015; 38:67-73.

23. Pongpech $\mathrm{P}, \quad$ Amornnopparattanakul S, Panapakdee $S$, et al. Antibacterial activity of carbapenem-based combinations againts multidrugresistant Acinetobacter baumannii. J Med Assoc Thai 2010; 93:161-171.

24. Keam S.J. Doripenem: a review of its use in the treatment of bacterial infections. Drugs 2008; 68:2021-2057.

25. Fernandez CF, Martinez ML, Conejo MC, Ayala JA, Perea EJ, Pascual A. Relationship between $\beta$ lactamase production, outer membrane protein and penicillin-binding protein profiles on the activity of carbapenems against clinical isolates of Acinetobacter baumannii. J Antimicrob Chemother. 2003; 51:565-574.

26. Gerner-Smidt P, Tjernberg I, Ursing J. Reliability of phenotypic tests for identification of Acinetobacter species. J Clin Microbiol 1991; 29:277-282.

27. Viehman JA, Nguyen MH, Doi Y. Treatment Options for Carbapenem-Resistant and Extensively Drug-Resistant Acinetobacter baumannii Infections. HHS Public Access. Drugs 2014; 74:1315-1333.

28. Falagas ME, Lourida $P$, Poulikakos $P$, Rafailidis PI, Tansarli GS. Antibiotic treatment of infections due to carbapenem-resistant Enterobacteriaceae:
Systematic evaluation of the available evidence. Antimicrob Agents Chemother 2014; 58:654-663.

29. Ghafur A, Devarajan V, Raja T, et al. Monotherapy versus combination therapy against carbapenemresistant Gram-negative bacteria: A retrospective observational study. Indian J Cancer 2016; 53: 592594.

30. Saiman L. Clinical utility of synergy testing for multi drug resistant Pseudomonas aeruginosa isolated from patients with cystic fibrosis: the motion for. Paediatr Respir Rev 2007; 8:249-255. 